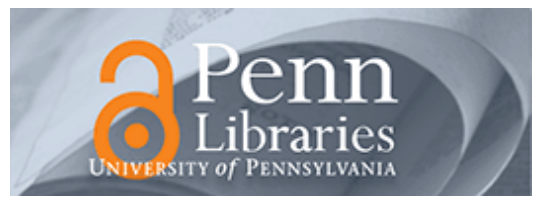

University of Pennsylvania

ScholarlyCommons

Finance Papers

Wharton Faculty Research

2008

\title{
An Equilibrium Guide to Designing Affine Pricing Models
}

Bjørn Eraker

Ivan Shaliastovich

University of Pennsylvania

Follow this and additional works at: https://repository.upenn.edu/fnce_papers

Part of the Finance Commons, and the Finance and Financial Management Commons

\section{Recommended Citation}

Eraker, B., \& Shaliastovich, I. (2008). An Equilibrium Guide to Designing Affine Pricing Models.

Mathematical Finance, 18 (4), 519-543. http://dx.doi.org/10.1111/j.1467-9965.2008.00346.x

At the time of publication, author Ivan Shaliastovich was affiliated with Duke University. Currently, he is a faculty member at the Wharton School at the University of Pennsylvania.

This paper is posted at ScholarlyCommons. https://repository.upenn.edu/fnce_papers/307

For more information, please contact repository@pobox.upenn.edu. 


\title{
An Equilibrium Guide to Designing Affine Pricing Models
}

\begin{abstract}
The paper examines equilibrium models based on Epstein-Zin preferences in a framework in which exogenous state variables follow affine jump diffusion processes. A main insight is that the equilibrium asset prices can be computed using a standard machinery of affine asset pricing theory by imposing parametric restrictions on market prices of risk, determined inside the model by preference and model parameters. An appealing characteristic of the general equilibrium setup is that the state variables have an intuitive and testable interpretation as driving the consumption and dividend dynamics. We present a detailed example where large shocks (jumps) in consumption volatility translate into negative jumps in equilibrium prices of the assets as agents demand a higher premium to compensate for higher risks. This endogenous "leverage effect," which is purely an equilibrium outcome in the economy, leads to significant premiums for out-of-the-money put options. Our model is thus able to produce an equilibrium "volatility smirk," which realistically mimics that observed for index options.

Disciplines

Finance | Finance and Financial Management

\section{Comments}

At the time of publication, author Ivan Shaliastovich was affiliated with Duke University. Currently, he is a faculty member at the Wharton School at the University of Pennsylvania.
\end{abstract}




\title{
An Equilibrium Guide to Designing Affine Pricing Models
}

\author{
Bjørn Eraker and Ivan Shaliastovich *
}

Duke University

\begin{abstract}
We examine equilibrium models based on Epstein-Zin preferences in a framework where exogenous state variables which drive consumption and dividend dynamics follow affine jump diffusion processes. Equilibrium asset prices can be computed using a standard machinery of affine asset pricing theory by imposing parametric restrictions on market prices of risk, determined by preference and model parameters. We present a detailed example where large shocks (jumps) in consumption volatility translate into negative jumps in equilibrium prices of the assets. This endogenous "leverage effect" leads to significant premiums for out-of-the-money put options. Our model is thus able to produce an equilibrium "volatility smirk" which realistically mimics that observed for index options.
\end{abstract}

KEY WORDS: Epstein-Zin preferences, affine asset pricing model, general equilibrium, option pricing

${ }^{*}$ We thank two anonymous referees and the associate editor for valuable comments. We have also benefited from discussions with Ravi Bansal, Tim Bollerslev, Xin Huang, George Tauchen, Stanley Zin, and seminar participants at University of Washington, the Lijiang Mathematical Finance workshop and the Duke Financial Econometrics workshop. Correspondence: bjorn.eraker@duke.edu and ivan.shaliastovich@duke.edu 


\section{Introduction}

A cornerstone of modern finance, no-arbitrage models are routinely applied to price basic securities such as stocks and bonds as well as derivative assets. No-arbitrage models place very few restrictions on the behavior of asset prices. Indeed, no-arbitrage models say (almost) nothing about the relationship between the assumed, objective probability law of the "state variables" in the model, and the arbitrage-induced "riskneutral" measure used for pricing. This is convenient from the point of view of practitioners who wish to maintain an infinite number of degrees of freedom in adjusting no-arbitrage models to observed asset prices. It is inconvenient and non-informative to academics who wish to design asset pricing models to study the dynamics of financial markets to learn about such things as market efficiency, investors' risk aversion, and the link between the macro economy and financial market prices.

In this paper we describe a consumption-based general equilibrium framework for designing affine asset pricing models when the representative agent is endowed with Epstein-Zin preferences over intermediate consumption and wealth (see Epstein and Zin 1989), and the underlying state variables follow a multivariate affine jump diffusion. The main message of the paper is that we can proceed to price stocks, bonds and derivatives by using a standard machinery of affine no-arbitrage models, under the conditions that 1) the market prices of risk are explicit functions of the preference parameters, and 2) state variables relate to the movements in aggregate consumption. We show that bond and stock prices are approximately exponential affine in state variables. We provide explicit expressions for the market prices of risk which depend on exogenous dynamics as well as preference parameters.

The Epstein-Zin preferences are crucial for our analysis because standard CRRA (power utility of consumption) preference structure implies that market prices of risk are zero for all shocks other than the immediate news to aggregate consumption. By contrast, the Epstein-Zin recursive utility function delivers non-zero market prices of risk for factors that are not directly related to consumption innovations. Thus, the Epstein-Zin framework offers important additional insights into why factors other than consumption shocks may be priced in asset markets.

We verify that market risk prices associated with small shocks (Wiener processes) have a standard linear form known from the no-arbitrage literature. This result is not surprising, and similar representations have been shown in discrete time models, such as those in Bansal and Yaron (2004) and Tauchen (2005). The linear market

price of risk representation implies that state variables follow affine jump diffusions under both the objective and the risk neutral probability measures.

While standard no-arbitrage models offer no guide in specifying a link between the objective and risk-neutral measures for discontinuous jumps, our framework provides an explicit formula for connecting the two measures. Specifically, we show that 
the risk-neutral jump intensities as well as the risk-neutral jump size distributions are obtained through a simple scalar adjustment of the arrival intensities and jump size distributions under the objective measure. In an example application, we show that both the jump arrival intensity and jump sizes are larger under the risk-neutral measure. The differences increase in the level of risk aversion of the representative agent.

In illustrating our approach, we present a detailed example model where aggregate consumption and dividend processes exhibit stochastic volatility. The volatility process, which we assume affects both dividend and consumption growth, follows a mean reverting process where shocks may be continuous (Wiener), discontinuous (compound Poisson), or both. Our model generates a negative correlation between shocks to the volatility process and the equilibrium stock prices. This correlation approaches negative one when the jumps dominate the variation in the volatility, and is different under the objective and risk-neutral distributions. No-arbitrage models to date have assumed that the negative volatility/stock price correlation is exogenously determined and identical under the two measures.

We study the equilibrium impact of volatility shocks on theoretical option prices in our model. Theoretical option prices are computed through the Fourier inversion technique of Lewis (2000), adapted to our setting with random, equilibrium-determined interest rates. The model produces several interesting stylized facts about options. The implied volatilities computed in our model tend to mimic those observed empirically in that the implied volatility is $U$ shaped, and with significantly higher prices for out-of-the-money puts. Low levels of risk aversion, conversely, produce a flatter volatility smile. This effect is not present in a model with CRRA utility, which tends to produce a reversed pattern in the implied volatility with relatively higher prices for ITM puts than OTM puts. The large impact of volatility shocks on OTM put options is related to two facts. First, the equilibrium stock price process is heavily influenced by the possibility of sudden increases (jumps) in economic uncertainty even under relatively modest levels of risk aversion. This generates large (negative) price jumps in the physical probability law of the stock price. Second, the adjustment of the physical probability law into the risk-neutral one implies an increase in both volatility jump arrival intensity, as well as the average sizes of the jumps. These risk adjustments are only present under the full Epstein-Zin preference model, and no such adjustment takes place for CRRA utility as shocks in the volatility are not explicitly correlated with the immediate innovations into the consumption growth.

Our paper is connected to the extant literature in several ways. Bansal and Yaron (2004) introduce the idea of long run risks and show that persistence in state variables coupled with an Epstein-Zin based equilibrium pricing kernel magnifies risk premiums relative to i.i.d. economies. Chen (2006) provides exact solutions (up to a system of nonlinear equations) in a continuous time setting when the state variable follows a time-homogeneous Markov chain process. Aase (2002) studies time-additive 
equilibrium with general utility under jump processes. Shaliastovich and Tauchen (2006) study equilibrium under subordinated Levy processes. Eraker (2006) examines a similar modeling environment in discrete time and studies example models for pricing stocks and bonds. Our paper generalizes most of the previous works, which are based on conditionally normal processes, to general affine processes. The advantage of continuous time approach in the current paper is that analytical tractability allows specific formulaes for market prices of risks, risk-neutral dynamics, etc. to be developed.

A number of papers have examined the implications of stochastic volatility and jump on option prices. Early examples include Hull and White (1987), Heston (1993), Bates (1996, 2000), Bakshi, Cao and Chen (1997), and Duffie, Pan and Singleton (2000). Madan, Carr and Chang (1998) and Carr et al. (2003) examine stochastic volatility models driven by subordinated Levy processes. Option pricing under recursive preferences has been studied by Liu, Pan and Wang (2005), Garcia, Luger and Renault (2003) and Benzoni, Collin-Dufresne and Goldstein (2005). Liu, Pan and Wang (2005) argue that Epstein-Zin preferences cannot explain the high valuations of OTM put options in their i.i.d economy and argue that the results are similar to results under CRRA utility. They conclude that a model in which investors do not know the true probability of a crash and exhibit uncertainty aversion is needed to explain high OTM put options. Benzoni, Collin-Dufresne and Goldstein (2005) show that Epstein-Zin preferences generate high valuations for OTM puts if the economy is not i.i.d, and expected consumption growth exhibits persistence along the lines of Bansal and Yaron (2004). Unlike this paper, our example application focuses on stochastic volatility as the driving force behind fat tailed return distributions and option premiums.

The remainder of this paper is organized as follows. Section 2 discusses the specification of the Epstein-Zin preferences and exogenous state variables and derives the equilibrium pricing kernel in the economy. In section 3 we discuss pricing of assets with various payoffs, including dividend-paying stocks and equity options. In section 4 we present an example model and examine the equilibrium stock price process and the implications for equity option prices computed in the model. Section 5 concludes.

\section{Model}

We start with a discrete time formulation of the real endowment economy where the representative agent's preferences over the uncertain consumption stream $C_{t}$ can be described by a recursive utility function of Epstein and Zin (1989) and Weil (1989):

$$
U_{t}=\left[(1-\delta) C_{t}^{\frac{1-\gamma}{\theta}}+\delta\left(E_{t} U_{t+1}^{1-\gamma}\right)^{\frac{1}{\theta}}\right]^{\frac{\theta}{1-\gamma}} .
$$


The representative agent's preferences are thus characterized by a subjective discount factor $\delta$, the intertemporal elasticity of substitution (IES) $\psi$ and the local risk aversion coefficient $\gamma . E_{t}$ denotes the standard expectation operator conditional on the information available to the agent in period $t$, and for notational convenience we set

$$
\theta=\frac{1-\gamma}{1-\frac{1}{\psi}}
$$

Notably, when the risk aversion coefficient is equal to the reciprocal of the IES, (equivalently, $\theta=1$ ), the preferences collapse to the familiar power utility case with risk aversion parameter $\gamma=\frac{1}{\psi}$. The novel and appealing characteristic of the generalized preferences is that they break the tight link between $\gamma$ and $\psi$ and allow to capture the agent's preference for the timing of the resolution of uncertainty. In the long run risks literature (see Bansal 2007 for a survey), risk aversion is larger than the reciprocal of the IES, $\gamma>1 / \psi$, that is, agents prefer early resolution of uncertainty. This ensures that the compensations for risks are of the right sign and quantitatively important. ${ }^{1}$

While one can view the recursive preferences in (2.1) as an important and economically appealing generalization of the standard, constant relative risk aversion expected utility, it is also possible to provide alternative interpretations of the recursion via concerns for risk and robustness to model misspecification. Tallarini (2000) enhances the value function of the standard log-utility agent by a risk sensitivity operator, which is mathematically equivalent to Epstein-Zin preferences (2.1) with IES and $\gamma$ equal to one. Hansen and Sargent (2006) and Barillas, Hansen and Sargent (2006) , on the other hand, re-interpret the recursion in Epstein-Zin preferences as an endogenous risk compensation for agent's distrust of model uncertainty and desire for robustness against the worst-case scenario. Finally, Maenhout (2004) shows that the behavior of the expected utility agent with a constant relative risk aversion and homothetic preference for robustness is observationally equivalent to that of an investor with a stochastic differential utility of Duffie and Epstein (1992), with an enhanced risk aversion coefficient. Thus, Epstein-Zin preferences provide important and interesting extensions of standard economic analysis which allow to examine different aspects of agent's attitude towards the underlying uncertainty in the economy.

In discrete time, the Epstein-Zin (EZ) preference structure leads to the following Euler equation

$$
E_{t}\left[\delta^{\theta}\left(\frac{C_{t+1}}{C_{t}}\right)^{-\frac{\theta}{\psi}} R_{c, t+1}^{-(1-\theta)} R_{i, t+1}\right]=1,
$$

where $R_{c, t}$ is the return on the aggregate wealth portfolio which pays consumption as its dividends and $R_{i, t}$ is the return on an arbitrary asset available to the investor. For analytical convenience, we choose the discrete time Euler equation in (2.2) as a starting point of our analysis despite the existence of a continuous time analogue pref- 
erence structure studied in Duffie and Epstein (1992), Schroder and Skiadas (1999), among others.

Notice that the discrete time recursion (2.1), or its continuous equivalent which we develop later in the paper, makes the pricing kernel non-affine if the log return on aggregate wealth $\ln R_{c, t}$ is non-linear. To maintain analytical tractability, therefore, we follow Campbell and Shiller (1988), Campbell (1993) and Bansal and Yaron (2004), among others, to linearize the model ${ }^{2}$.

Specifically, the discrete time continuously compounded (log) return $\ln R_{t}$ on any asset with price $P_{t}$ and dividend level $D_{t}$ can be expressed as

$$
\begin{aligned}
\ln R_{t+1} & =\ln \frac{P_{t+1}+D_{t+1}}{P_{t}} \\
& \equiv \ln \left(e^{\ln \frac{P_{t+1}}{D_{t+1}}}+1\right)-\ln \frac{P_{t}}{D_{t}}+\ln \frac{D_{t+1}}{D_{t}} .
\end{aligned}
$$

Log-linearize the first summand around the mean log price-dividend ratio to obtain

$$
\ln R_{t+1} \approx k_{0}+k_{1} v_{t+1}-v_{t}+\Delta \ln D_{t+1},
$$

where $\Delta \ln D_{t+1}=\ln \frac{D_{t+1}}{D_{t}}$ and $v_{t}=\ln P_{t}-\ln D_{t}$. The approximation error is given by the second-order Taylor residual; for notational ease, we suppress it in a subsequent discussion and treat the approximated returns as exact. Campbell, Lo and Mackinlay (1997) find that the absolute approximation errors for the mean and standard deviation of US returns over the period 1926 to 1994 is $-0.17 \%$ and $0.26 \%$, respectively. Bansal, Kiku and Yaron (2006) prove that if the IES parameter $\psi$ is equal to one, the approximation error for the model-implied equilibrium returns is exactly zero, while for $\psi>1$ they find the relative approximation errors for the model-implied mean and standard deviation of the log price-consumption ratio being less than $1 \%$. Thus, while linearization of returns facilitates the analytical tractability of the model, we believe it does not have any first-order effects on the asset prices.

The constants $k_{0}$ and $k_{1}$ depend on the mean $\log$ valuation ratio $E\left(v_{t}\right)$ :

$$
\begin{aligned}
k_{1} & =\frac{e^{E\left(v_{t}\right)}}{1+e^{E\left(v_{t}\right)}}, \\
k_{0} & =-\ln \left[\left(1-k_{1}\right)^{1-k_{1}} k_{1}^{k_{1}}\right] .
\end{aligned}
$$

In equilibrium, the model-implied mean price-dividend ratio $E\left(v_{t}\right)$ should be consistent with the linearization coefficients $k_{0}$ and $k_{1}$. We show that this imposes a non-linear constraint on $k_{1}$, which can be solved recursively given the parameters of the model.

While the approximation (2.3) applies in discrete time, in Appendix A we show that its continuous time counterpart can be consistently defined in the following way:

$$
d \ln R_{t}=k_{0} d t+k_{1} d v_{t}-\left(1-k_{1}\right) v_{t} d t+d \ln D_{t},
$$


where $d v_{t}$ and $d \ln D_{t}$ are the instantaneous changes in log price-dividend ratio and $\log$ dividend level, respectively. Parameters $k_{0}$ and $k_{1}$ can thus be interpreted as linearization coefficients that are relevant over a unit of time.

The log-linearization of return (2.6) is a key to derive a continuous time counterpart to the standard discrete time formulation of the economy. Indeed, in Section 2.2 we use it to explicitly characterize the continuous time equivalent of the Euler equation in (2.2), which enables us to solve for the pricing kernel and equilibrium asset prices in terms of the underlying state variables $X_{t}$. We provide a clear interpretation of $X_{t}$ as a set of common economic fundamentals which affect the dynamics of consumption growth as well as dividends of individual assets. We turn to the specification of these variables in the next section.

\subsection{State Variables}

We follow here the presentation of Duffie, Pan and Singleton (2000) and assume that there is a set of $n$ state variables in the economy which follow the affine jump diffusion process. Specifically, we fix the probability space $\{\Omega, \mathcal{F}, \mathcal{P}\}$ and the information filtration $\mathcal{F}_{t}$, and suppose that $X_{t}$ is a Markov process in some state space $\mathcal{D} \subseteq \mathbb{R}^{n}$ with a stochastic differential equation representation

$$
d X_{t}=\mu\left(X_{t}\right) d t+\Sigma\left(X_{t}\right) d W_{t}+\xi_{t} \cdot d N_{t}
$$

$W_{t}$ is an $\mathcal{F}_{t}$ adapted Brownian motion in $\mathbb{R}^{n}$. The term $\xi_{t} \cdot d N_{t}$ (element-by-element multiplication) captures conditionally independent jumps arriving with intensity $l\left(X_{t}\right)$ and jump size distribution $\xi_{t}$ on $\mathcal{D}$. Intuitively, conditional on the path of $X$, the jump arrivals are the jump times of the Poisson distribution with possibly time-varying intensity $l\left(X_{t}\right)$. We further assume that jump sizes $\xi$ are i.i.d. in time and crosssectionally; their distribution is specified through the "jump transform" (individual generating function) $\varrho: \mathbb{C} \rightarrow \mathbb{C}$,

$$
E e^{u \xi}=\varrho(u)
$$

With a slight abuse of notation, we will sometimes evaluate $\varrho($.$) at a vector argu-$ ment, which we take to mean a stack of element-by-element application of the jump transform. We assume that the moment-generating function of $\xi$ exists such that $\varrho$ is well defined for both complex and real arguments on some region of the complex plane. This is a somewhat restrictive assumption which rules out certain heavy tailed distributions including power-law ones. 
We further impose an affine structure on the drift, diffusion and intensity functions:

$$
\begin{aligned}
\mu\left(X_{t}\right) & =\mathcal{M}+\mathcal{K} X_{t}, \\
\Sigma\left(X_{t}\right) \Sigma\left(X_{t}\right)^{\prime} & =h+\sum_{i} H_{i} X_{t, i}, \\
l\left(X_{t}\right) & =l_{0}+l_{1} X_{t},
\end{aligned}
$$

for $(\mathcal{M}, \mathcal{K}) \in \mathbb{R}^{n} \times \mathbb{R}^{n \times n},(h, H) \in \mathbb{R}^{n \times n} \times \mathbb{R}^{n \times n \times n},\left(l_{0}, l_{1}\right) \in \mathbb{R}^{n} \times \mathbb{R}^{n \times n}$. For $X$ to be well defined, there are additional joint restrictions on the parameters of the model, which are addressed in Duffie and Kan (1996).

We assume that the log consumption and dividend growth rates are linear in the states:

$$
\begin{aligned}
d \ln C_{t} & =\delta_{c}^{\prime} d X_{t}, \\
d \ln D_{t} & =\delta_{d}^{\prime} d X_{t} .
\end{aligned}
$$

We typically structure the state variables so that the consumption growth is the first factor, while the dividend growth rate is the last one, so $\delta_{c}$ and $\delta_{d}$ become selection vectors $(1,0,0, \ldots)$ and $(\ldots, 0,0,1)$, respectively. This model setup follows Eraker (2006).

\subsection{Equilibrium}

In the following we explicitly derive the equilibrium pricing kernel in our economy in continuous time. Our strategy is to translate the Euler condition (2.2) in discrete time into the martingale restriction in continuous time, relying on the continuous time limit of $\log$ return defined in (2.6). Setting $R_{i, t+1}=R_{c, t+1}$ in the Euler equation (2.2), we first solve for the equilibrium return on the aggregate wealth portfolio. This enables us to characterize the pricing kernel and the risk-neutral probability measure, which can be used to price any asset in the economy.

Our economy is set up such that each asset pays a random dividend continuously in time. To convert the continuous time dividend and price process into a discrete time return, we define the discrete time return to be the return on a portfolio which re-invests the continuously paid dividends. The discrete time return on this asset is just the aggregate continuous time log return,

$$
\int_{t}^{t+1} d \ln R_{i, s}
$$

The Euler equation (2.2) becomes

$$
E_{t} \exp \left[\ln \frac{M_{t+1}}{M_{t}}+\int_{t}^{t+1} d \ln R_{i, s}\right]=1 .
$$


where $M_{t}$ is the marginal utility of the agents, whose log-increments in discrete time are given by

$$
\ln M_{t+1}-\ln M_{t}=\theta \ln \delta-\frac{\theta}{\psi}\left(\ln C_{t+1}-\ln C_{t}\right)-(1-\theta) \int_{t}^{t+1} d \ln R_{c, s} .
$$

Thus, we can consistently define the continuous time dynamics of the pricing kernel in the following way:

$$
d \ln M_{t}=\theta \ln \delta d t-\frac{\theta}{\psi} d \ln C_{t}-(1-\theta) d \ln R_{c, t} .
$$

Note that the marginal utility $M_{t}$ depends on the equilibrium return on the consumption asset $R_{c, t}$, which is itself endogenous to the model. To obtain explicit solutions in terms of the fundamental state variables, we first conjecture that the log price-consumption ratio $v_{t}$ is affine in $X_{t}$ :

$$
v_{t}=A+B^{\prime} X_{t} .
$$

Setting $R_{i, t}=R_{c, t}$ in the Euler equation (2.8), we can derive the loadings $A$ and $B$, which verifies our conjecture for the equilibrium solution to the value of the wealth portfolio. For this purpose, define

$$
\begin{aligned}
\ln \frac{Z_{t+1}}{Z_{t}} & =\ln \frac{M_{t+1}}{M_{t}}+\int_{t}^{t+1} d \ln R_{c, s} \\
& =\theta \ln \delta-\frac{\theta}{\psi}\left(\ln C_{t+1}-\ln C_{t}\right)+\theta \int_{t}^{t+1} d \ln R_{c, s} .
\end{aligned}
$$

Using the continuous time dynamics of the discount factor in (2.10) and state variables in (2.23), expression for linearized log returns in (2.6) and conjecture for the log price-consumption ratio (2.11), we can express the evolution of $\ln Z_{t}$ in continuous time in the following way:

$$
\begin{aligned}
d \ln Z_{t}= & \theta \ln \delta d t-\frac{\theta}{\psi} d \ln C_{t}+\theta d \ln R_{c, t} \\
= & {\left[\theta \ln \delta+\chi^{\prime}\left(\mathcal{M}+\mathcal{K} X_{t}\right)+\theta k_{0}-\theta\left(1-k_{1}\right)\left(A+B^{\prime} X_{t}\right)\right] d t } \\
& +\chi^{\prime} \Sigma\left(X_{t}\right) d W_{t}+\chi^{\prime}\left(\xi_{t} \cdot d N_{t}\right),
\end{aligned}
$$

where $\chi=\theta\left(\left(1-\frac{1}{\psi}\right) \delta_{c}+k_{1} B\right)$.

Next, from (2.8) it follows that $Z_{t}=E_{t} Z_{t+1}$, so that $Z_{t}$ is a martingale. In continuous time, it implies that its drift must be equal to 0. Using Ito's lemma, we obtain

$$
\begin{aligned}
\theta \ln \delta & +\chi^{\prime}\left(\mathcal{M}+\mathcal{K} X_{t}\right)+\theta k_{0}-\theta\left(1-k_{1}\right)\left(A+B^{\prime} X_{t}\right) \\
& +\frac{1}{2} \chi^{\prime} \Sigma\left(X_{t}\right) \Sigma\left(X_{t}\right)^{\prime} \chi+(\varrho(\chi)-1)^{\prime} l\left(X_{t}\right)=0
\end{aligned}
$$


Matching the coefficients on a constant and $X_{t}$, we obtain the following equations for $A$ and $B$ :

$$
\begin{aligned}
& 0=\mathcal{K}^{\prime} \chi-\theta\left(1-k_{1}\right) B+\frac{1}{2} \chi^{\prime} H \chi+l_{1}^{\prime}(\varrho(\chi)-1), \\
& 0=\theta\left(\ln \delta+k_{0}-\left(1-k_{1}\right) A\right)+\mathcal{M}^{\prime} \chi+\frac{1}{2} \chi^{\prime} h \chi+l_{0}^{\prime}(\varrho(\chi)-1) .
\end{aligned}
$$

In general, these equations can yield multiple solutions to $A$ and $B$. In our numerical example, we generalize the criterion in Tauchen (2005) and select the root which ensures the non-explosiveness of the system as the contributions of stochastic volatility and jump components converge to zero. An alternative approach is to choose an "economically reasonable" solution which responds intuitively to model and preference parameters. We will provide more discussion in the empirical section of the paper.

Similar to Bansal, Kiku and Yaron (2006), we solve for the linearization constants $k_{0}$ and $k_{1}$ as part of the equilibrium solution of the model. From (2.11)

$$
E\left(v_{t}\right)=A+B^{\prime} \mu_{X},
$$

where $\mu_{X}$ is the vector with $i$ th component

$$
\mu_{X, i}= \begin{cases}E\left(X_{i}\right) & \text { if } E\left(X_{i}\right) \text { exists } \\ 0 & \text { otherwise }\end{cases}
$$

Expanding $k_{0}$ in terms of $k_{1}$ we can show that

$$
\begin{aligned}
k_{0}+\left(k_{1}-1\right) A & =k_{0}-\left(1-k_{1}\right)\left(E\left(v_{t}\right)-B^{\prime} \mu_{X}\right) \\
& =-\ln k_{1}+\left(1-k_{1}\right) B^{\prime} \mu_{X} .
\end{aligned}
$$

Plugging this expression into (2.12), we obtain that the linearization coefficient $k_{1}$ satisfies the following non-linear equation:

$$
\theta \ln k_{1}=\theta\left(\ln \delta+\left(1-k_{1}\right) B^{\prime} \mu_{X}\right)+\mathcal{M}^{\prime} \chi+\frac{1}{2} \chi^{\prime} h \chi+l_{0}^{\prime}(\varrho(\chi)-1) .
$$

Given the parameters of the model, we numerically iterate on $k_{1}$ in the formula above starting from the initial value $\delta$, which is the exact the solution for $k_{1}$ when $\psi=1$. For the parameter values we consider, the algorithm converges very fast, in $2-5$ iterations.

Using the equilibrium solution to the return on wealth portfolio, the evolution of the log pricing kernel can now be written in terms of the economic fundamentals:

$$
\begin{aligned}
d \ln M_{t} & =\theta \ln \delta d t-\frac{\theta}{\psi} d \ln C_{t}+(\theta-1) d \ln R_{c, t} \\
& =\left(\theta \ln \delta-(\theta-1) \ln k_{1}+(\theta-1)\left(k_{1}-1\right) B^{\prime}\left(X_{t}-\mu_{X}\right)\right) d t-\lambda^{\prime} d X_{t},
\end{aligned}
$$


where

$$
\lambda=\gamma \delta_{c}+(1-\theta) k_{1} B
$$

This equation offers some key insights into the difference between Epstein-Zin and CRRA preferences. Without loss of generality, we may assume that the first statevariable is the $(\log )$ consumption, in which case the selection vector $\delta_{c}=(1,0,0 \ldots)$. Now if $\gamma=1 / \psi \Leftrightarrow \theta=1$, the Epstein-Zin preferences collapse into the familiar CRRA case, and thus $\lambda=(\gamma, 0,0, \ldots)$. As we show below, $\lambda$ determines the market prices of risk for the different components of $X$, such that if $\lambda_{i}=0$, then innovations into $X_{i}$ are not priced. Therefore, while consumption is the only priced factor in CRRA utility models, Epstein-Zin preferences usually imply that all state variables are priced, since $\theta \neq 1$ and $B$ and $k_{1}$ are different from zero.

From the expression for the pricing kernel (2.17), we obtain that the instantaneous risk-free rate $r_{t}$ is affine in $X_{t}$,

$$
r_{t}=\Phi_{0}+\Phi_{1}^{\prime} X_{t}
$$

As $M_{t} e^{\int_{0}^{t} r(s) d s}$ is a martingale, we use Ito's lemma to obtain

$$
\begin{aligned}
\Phi_{1}= & (1-\theta)\left(k_{1}-1\right) B+\mathcal{K}^{\prime} \lambda-\frac{1}{2} \lambda^{\prime} H \lambda-l_{1}^{\prime}(\varrho(-\lambda)-1), \\
\Phi_{0}= & -\theta \ln \delta+(\theta-1)\left(\ln k_{1}+\left(k_{1}-1\right) B^{\prime} \mu_{X}\right)+\mathcal{M}^{\prime} \lambda \\
& -\frac{1}{2} \lambda^{\prime} h \lambda-l_{0}^{\prime}(\varrho(-\lambda)-1) .
\end{aligned}
$$

Thus, we can substitute the short rate to express the evolution of the discount factor in the following way:

$$
\frac{d M_{t}}{M_{t-}}=-r_{t} d t-\Lambda_{t}^{\prime} d W_{t}-\sum_{i}\left[\left(1-e^{-\lambda^{i} \xi_{t}^{i}}\right) d N_{t}^{i}-\left(1-\varrho\left(-\lambda^{i}\right)\right) l^{i}\left(X_{t}\right) d t\right],
$$

where superscript $i$ denotes the $i$ th element in the vector, and $\Lambda_{t}$ is defined by

$$
\Lambda_{t}=\Sigma\left(X_{t}\right)^{\prime} \lambda
$$

The vector $\Lambda_{t}$ is related to the price of jump risk of size $\xi$ in $i$ th state variable and literarily is the price of Brownian motion risk. The following theorem is a slight generalization of Proposition 5 in Duffie, Pan and Singleton (2000) which describes the evolution of the system under the risk-neutral measure.

Theorem 2.1. Under the risk-neutral measure $Q$ induced by the discount factor $M_{t}$ the state variables follow

$$
d X_{t}=\left(\mathcal{M}^{Q}+\mathcal{K}^{Q} X_{t}\right) d t+\Sigma\left(X_{t}\right) d W_{t}^{Q}+\xi_{t}^{Q} \cdot d N_{t}^{Q}
$$


where

$$
\begin{aligned}
\mathcal{M}^{Q} & =\mathcal{M}-h \lambda \\
\mathcal{K}^{Q} & =\mathcal{K}-H \lambda \\
d W_{t}^{Q} & =d W_{t}+\Lambda_{t} d t
\end{aligned}
$$

defines a Brownian motion under the risk-neutral measure.

The $Q$ jump-arrival intensity is given by

$$
l_{t}^{Q}=l_{t} \cdot \varrho(-\lambda)
$$

The $Q$ jump-size density is characterized by its Laplace transform $\varrho^{Q}: \mathbb{C}^{n} \rightarrow \mathbb{C}^{n}$

$$
\varrho^{Q}(u)=E^{Q} e^{u \xi}=\varrho(u-\lambda) \cdot / \varrho(-\lambda) .
$$

Notice that if $\lambda_{i}=0$, there is no difference in the jump measures and both market prices of diffusion and jump risks are zero. This pinpoints the importance of the parameters $\lambda$ in generating risk premia in our model.

The jump intensity is greater (smaller) under the equivalent measure $Q$ whenever $\lambda$ is negative (positive). The mean and standard deviation of jump size are greater under the risk-neutral than objective measure when $\varrho\left(-\lambda_{i}\right) \in(0,1)$ and smaller if $\varrho\left(-\lambda_{i}\right)>1$, as

$$
\begin{aligned}
E^{Q} \xi & =E(\xi) / \varrho\left(-\lambda_{i}\right), \\
\operatorname{Std}_{Q}\left(\xi_{i}\right) & =\operatorname{Std}_{P}\left(\xi_{i}\right) \varrho\left(-\lambda_{i}\right)^{-\frac{1}{2}} .
\end{aligned}
$$

The following reward-to-risk ratio illustrates the equilibrium rewards for jump risks,

$$
\Lambda_{i}^{J} \equiv \frac{E \xi_{i}-E^{Q} \xi_{i}}{\operatorname{Std}\left(\xi_{i}\right)}=\frac{E \xi_{i}}{\operatorname{Std}\left(\xi_{i}\right)}\left(1-\frac{1}{\varrho\left(-\lambda_{i}\right)}\right)
$$

It is somewhat misleading, although tempting, to coin this measure a market price of jump risk. Jump risks are characterized, and thus priced, not only according to their mean and standard deviations, but also higher order moments.

To build more intuition about the risk-neutral adjustment to the overall density of jump amplitudes, let us examine a particular case when the jump size $\xi$ belong to an infinitely divisible class of distributions with finite variation Lévy measure, which includes Gaussian, gamma, $\alpha$-stable and tempered stable, compound Poisson distribution and others. In particular we can write down the moment-generating function of jumps under the physical measure as

$$
\varrho(u)=e^{\mu u+\frac{1}{2} \sigma^{2} u^{2}+\int_{R}\left(e^{u x}-1\right) \omega(d x)},
$$


for certain $\mu$ and $\sigma$ and positive Radon measure $\omega .{ }^{3}$ It is easy to see that if it exists, the risk-neutral distribution of jump sizes will remain infinitely-divisible, with the following parameters:

$$
\begin{aligned}
\mu^{Q} & =\mu-\lambda \sigma^{2}, \\
\sigma^{Q} & =\sigma, \\
\omega^{Q}(d x) & =e^{-\lambda x} \omega(d x) .
\end{aligned}
$$

For $\lambda<0$, the risk-neutral adjustment shifts the distribution of jump sizes to the right and fattens its tails. Therefore, the investors adjust their perception of large negative jump-news in the economy by making them higher on average and more extreme under the risk-adjusted probabilities, while the opposite happens if $\lambda>0$. For particular examples of the risk-neutral transformations of the jump size distribution, refer to Table 1.

[Table 1 about here.]

\section{$3 \quad$ General asset prices}

Consider the price of an asset which pays a continuous dividend stream $D_{s}, t<s<T$. The price of this asset (stock) is now obtained by taking the expectation under the risk-neutral measure of its discounted payoffs $D\left(X_{t}\right)$ :

$$
\begin{aligned}
P\left(\left\{D\left(X_{s}\right)\right\}_{s=t}^{T}\right) & =\int_{t}^{T} E_{t}\left(\frac{M_{s}}{M_{t}} D_{s}\right) d s \\
& \equiv \int_{t}^{T} E_{t}^{Q}\left(e^{-\int_{t}^{s} r(u) d u} D_{s}\right) d s
\end{aligned}
$$

Expiration date $T$ can also be infinity, provided that the price is finite.

To facilitate the computations, we follow Duffie, Pan and Singleton (2000) and compute a discounted characteristic function of $X_{t}$ under the risk-neutral measure:

$$
\varrho_{X}^{Q}\left(u, X_{t}, s\right)=E_{t}^{Q}\left(e^{-\int_{t}^{t+s} r(\tau) d \tau} e^{u^{\prime} X_{t+s}}\right) .
$$

for $u \in \mathbb{C}^{n}$.

Under appropriate technical regularity conditions (see Duffie, Pan and Singleton $(2000)), \varrho_{X}^{Q}$ is exponential affine in $X_{t}$,

$$
\varrho_{X}^{Q}\left(u, X_{t}, s\right)=e^{\alpha(s)+\beta(s)^{\prime} X_{t}}
$$


where $\alpha(s)$ and $\beta(s)$ satisfy complex-valued ordinary differential equations

$$
\begin{aligned}
& \dot{\beta}=-\Phi_{1}+\mathcal{K}^{Q^{\prime}} \beta+\frac{1}{2} \beta^{\prime} H \beta+l_{1}^{Q^{\prime}}\left(\varrho^{Q}(\beta)-1\right), \\
& \dot{\alpha}=-\Phi_{0}+\mathcal{M}^{Q^{\prime}} \beta+\frac{1}{2} \beta^{\prime} h \beta+l_{0}^{Q^{\prime}}\left(\varrho^{Q}(\beta)-1\right),
\end{aligned}
$$

subject to boundary conditions $\beta(0)=u, \alpha(0)=0$.

In particular, setting $u=0$ we immediately obtain that the yield on a discount bond with $s$ periods to maturity is given by,

$$
y\left(X_{t}, s\right)=-\frac{1}{s}\left(\alpha(s)+\beta(s)^{\prime} X_{t}\right)
$$

where $\alpha$ and $\beta$ solve the ODEs in (3.2) with boundary condition $\beta(0)=\alpha(0)=0$. Note that as the initial values for $\alpha$ and $\beta$ are real and the risk-neutral mgf of jump distribution is assumed to exist (i.e. take real values), the solution to the yield curve is guaranteed to be real as well.

\subsection{Dividend Paying Assets}

Consider an asset which dividend stream can be expressed as a linear function of the state variables,

$$
d \ln D_{t}=\delta_{d}^{\prime} d X_{t}
$$

From the discussion in the previous section, the price of an asset which pays a perpetual dividend $D_{t}$, if it exists, is given by

$$
\begin{aligned}
P_{t}\left(X_{t}\right) & =\int_{0}^{\infty} \varrho_{X}^{Q}\left(\delta_{d}, X_{t}, s\right) d s \\
& =\int_{0}^{\infty} e^{\alpha(s)+\beta(s)^{\prime} X_{t}} d s
\end{aligned}
$$

where $\alpha$ and $\beta$ satisfy the ODEs in (3.2) subject to $\beta(0)=\delta_{d}$ and $\alpha(0)=0$.

Equation (3.3) gives the exact equilibrium price-dividend ratio when the stock pays a perpetual dividend. To build more intuition about the model, we consider an approximate equilibrium solution which is obtained, as for the consumption asset, through the log-linearization of returns. It is straightforward to show that in this case, the equilibrium price-dividend ratio is exponential linear,

$$
P_{t}=D_{t} \exp \left(A_{d}+B_{d}^{\prime} X_{t}\right)
$$

where the coefficients $A_{d}$ and $B_{d}$ satisfy

$$
\mathcal{K}^{\prime} \chi_{d}+(\theta-1)\left(k_{1}-1\right) B+\left(k_{1, d}-1\right) B_{d}+\frac{1}{2} \chi_{d}^{\prime} H \chi_{d}+l_{1}^{\prime}\left[\rho\left(\chi_{d}\right)-1\right]=0
$$


and

$$
\begin{aligned}
\theta \ln \delta-(\theta-1)\left(\ln k_{1}+\left(k_{1}-1\right) B^{\prime} \mu_{X}\right) & -\left(\ln k_{1, d}+\left(k_{1, d}-1\right) B_{d}^{\prime} \mu_{X}\right) \\
& +\mathcal{M}^{\prime} \chi_{d}+\frac{1}{2} \chi_{d}^{\prime} h \chi_{d}+l_{0}\left[\rho\left(\chi_{d}\right)-1\right]=0
\end{aligned}
$$

for

$$
\chi_{d}=\delta_{d}+k_{1, d} B_{d}-\lambda,
$$

and $k_{1, d}$ is the log-linearization coefficient for a dividend return. As before, we can solve the equations above for $B_{d}$ and $k_{1, d}$, and then obtain an intercept $A_{d}$ from the chain of equalities

$$
\begin{aligned}
A_{d}+B_{d}^{\prime} \mu_{X} & =E \ln \frac{P_{t}}{D_{t}} \\
& =\ln \frac{k_{1, d}}{1-k_{1, d}} .
\end{aligned}
$$

The first equality follows from the conjectured solution for the price-dividend ratio, while the second one comes from the log-linearization procedure.

The main advantage of the formulae presented in this section is that we can obtain an exponential affine representation of the equilibrium stock price dynamics, subject to our log-linearization of returns. This facilitates the computations of option prices, as illustrated next.

\subsection{Option Pricing}

Lewis (2000) and Carr and Madan (1999) discuss methods for computing option prices from the characteristic function of the underlying stock price. In the following we adapt the formula in Lewis (2000) to our setting.

The price of a European call option is a function of the state variables $X_{t}$, strike price $K$ and maturity of an option $s$ :

$$
C\left(X_{t}, K, s\right)=E_{t}^{Q}\left[e^{-\int_{t}^{t+s} r_{\tau} d \tau}\left(e^{\ln P_{t+s}}-K\right)^{+}\right]
$$

Using the Parseval identity, we obtain that

$$
\begin{aligned}
C\left(X_{t}, K, s\right) & =E_{t}^{Q}\left[e^{-\int_{t}^{t+s} r_{\tau} d \tau}\left(e^{\ln P_{t+s}}-K\right)^{+}\right] \\
& =\frac{1}{2 \pi} E_{t}^{Q}\left[\int_{i z_{i}-\infty}^{i z_{i}+\infty} e^{-\int_{t}^{t+s} r_{\tau} d \tau} e^{-i z \ln P_{t+s}} \hat{w}(z) d z\right]
\end{aligned}
$$


where the generalized Fourier transform of the payoff function of the option $\hat{w}(z)$ is equal to,

$$
\begin{aligned}
\hat{w}(z) & =\int_{-\infty}^{\infty} e^{i z x}\left(e^{x}-K\right)^{+} d x \\
& =-\frac{K^{i z+1}}{z^{2}-i z},
\end{aligned}
$$

for $z_{i} \equiv \operatorname{Im}(z)>1$, and identical expression obtains for put options for $z_{i}<0$.

If a stock pays a single terminal dividend $D_{T}$ at some date $T>s>t$ or if we log-linearize the returns on a stock which pays dividend continuously, the equilibrium value of an asset will be linear in the state variables:

$$
\ln P_{t}=A_{d}+\left(B_{d}+\delta_{d}\right)^{\prime} X_{t} .
$$

Using a discounted characteristic function of state variables under the risk-neutral measure defined in (3.1), we can rewrite the expression for the option price in the following way:

$$
C\left(X_{t}, K, s\right)=-K \frac{1}{2 \pi} \int_{i z_{i}-\infty}^{i z_{i}+\infty} e^{-i z A_{d}} \varrho_{X}^{Q}\left(-z\left(B_{d}+\delta_{d}\right), X_{t}, s\right) \frac{K^{i z}}{z^{2}-i z} d z
$$

The integration in (3.10) is performed on the intersection of the strips $z_{i}>1$ for call option or $z_{i}<0$ for puts, and the one parallel to the real $z$-axis. Notice that (3.10) requires a single numerical integration, which is advantageous relative to the formulae in the extant literature (e.g. Heston 1993; Bates 1996; Duffie, Pan and Singleton 2000) which require two numerical integrations. In addition, the discounted characteristic function $\varrho_{X}^{Q}$ is known up to a system of ordinary differential equations (3.2), which depend on preference, cash flow parameters and maturity of an option, but do not involve strike price $K$. In this case, calculating option prices for a range of strikes is particularly convenient and fast.

\section{The Equilibrium Impact of Volatility Shocks}

In an application of our model, we consider an economy in which consumption, dividends and in the end asset prices, are influenced by a single state variable, which is the conditional volatility of consumption growth. To this end we assume that log aggregate consumption follows

$$
\begin{aligned}
\mathrm{d} \ln C_{t} & =\mu d t+\sqrt{V_{t}} d W_{c, t}, \\
\mathrm{~d} V_{t} & =\kappa_{V}\left(\bar{V}-V_{t}\right) d t+\sigma_{v} \sqrt{V_{t}} d W_{V, t}+\xi_{V} d N_{t}, \\
\xi_{V} & \sim G A\left(\nu, \mu_{V} / \nu\right), \\
l\left(V_{t}\right) & =l_{0}+l_{1} V_{t} .
\end{aligned}
$$


The volatility process $V_{t}$ is driven by the continuous Brownian motion $d W_{V, t}$ as well as discontinuous process $\xi_{V} d N_{t}$, whose arrival intensity is $l\left(V_{t}\right)$. Our assumption of Gamma distributed volatility jump sizes allows a fairly heavily tailed jump size distribution for small values of the scale parameter $\nu$.

The specification above encompasses a number of stochastic volatility models in the literature, including square root volatility model of Heston (1993), the exponential jump diffusion model of Duffie, Pan and Singleton (2000), Eraker (2004), among others. By removing the diffusion part, $\sigma_{v}=0$, the volatility dynamics reduces to the gamma OU process $\left(l_{1}=0\right.$ and $\left.\nu=1\right)$. For a detailed treatment of Non-Gaussian OU processes refer to Barndorff-Nielsen and Shephard (2001). Our specification of the consumption process is a simplification of the Bansal and Yaron (2004) model in that the expected log consumption growth is constant. In the Bansal and Yaron (2004) model, the expected consumption growth follows a mean-reverting AR(1) process. Fixing the expected growth rate in our model allows us to focus entirely on the equilibrium effects of stochastic volatility.

The first step in our analysis is to recover expressions for the coefficients $A$ and $B$ in eqns. (2.12) and (2.13). The loading on consumption growth is zero, while the "volatility factor loading" $B_{v}$ solves

$0=-\theta\left[\kappa_{v} k_{1}+\left(1-k_{1}\right)\right] B_{v}+\frac{1}{2} \theta^{2}\left(1-\frac{1}{\psi}\right)^{2}+\frac{1}{2} \theta^{2} k_{1}^{2} \sigma_{v}^{2} B_{v}^{2}+l_{1}\left[\left(1-B_{v} \theta k_{1} \mu_{v} / \nu\right)^{-\nu}-1\right]$.

This equation admits an explicit solution only in special cases. In particular, if there are no state-dependent volatility jumps, $l_{1}=0$, then $B_{v}$ solves the quadratic equation $a+b B_{v}+c B_{v}^{2}=0$ for $a=\theta^{2} \frac{1}{2}\left(1-\frac{1}{\psi}\right)^{2}, b=-\theta\left(\kappa_{v}+\left(1-k_{1}\right)\right), c=\theta^{2} \kappa_{1}^{2} \sigma_{v}^{2}$. Tauchen (2005) points out that square root processes for volatility generally produce two roots for $B_{v}$. However, if $\theta<0, b$ is positive, so that only the "right" root is non-explosive when a stochastic volatility parameter $\sigma_{v}$ converges to 0 . By including state-dependent volatility jumps, we generally have more than two roots. In the case where the volatility is driven by pure jumps $\left(\sigma_{v}=0\right)$ and volatility jumps are exponentially distributed $(\nu=1)$, we can recover another quadratic equation for $B_{v}$, and we can use a similar argument to select the non-explosive solution when the jump contribution is converging to zero. When $\sigma_{v}$ and $l_{1}$ are not zero, for reasonable parameter values we typically obtain two real solutions for $B_{v}$, and we choose the "right" root near the one implied by a quadratic equation above. 
Given the solution to $B_{v}$, we recover the dynamics of the state variables under the risk-neutral measure:

$$
\begin{aligned}
\mathrm{d} \ln C_{t} & =\left(\mu-\gamma V_{t}\right) d t+\sqrt{V_{t}} d W_{c, t}^{Q} \\
\mathrm{~d} V_{t} & =\kappa_{V}\left(\bar{V}-V_{t}\right) d t-\lambda_{v} \sigma_{v}^{2} V_{t} d t+\sigma_{v} \sqrt{V_{t}} d W_{V, t}^{Q}+\xi_{V}^{Q} d N_{t}^{Q} \\
\xi_{V}^{Q} & \sim G A\left(\nu, \frac{\mu_{v}}{\nu+\lambda_{v} \mu_{v}}\right) \\
l^{Q}\left(V_{t}\right) & =\left(1+\frac{\lambda_{v} \mu_{v}}{\nu}\right)^{-\nu} l\left(V_{t}\right),
\end{aligned}
$$

where $\lambda_{v}=(1-\theta) k_{1} B_{v}$ denotes the price of the variance shock.

This process is well defined whenever $\nu>\lambda_{v} \mu_{v}$, which places implicit restrictions on the permissible preference parameters. If this holds and the market price of volatility risk is negative, then both jump sizes and arrival intensity are greater under the risk-neutral measure,

$$
\begin{aligned}
E^{Q} \xi_{V} & >E^{P} \xi_{V}, \\
\operatorname{Var}^{Q} \xi_{V} & >\operatorname{Var}^{P} \xi_{V}, \\
l^{Q}\left(V_{t}\right) & >l\left(V_{t}\right) .
\end{aligned}
$$

In the case of exponentially distributed jump sizes, $\nu=1$, and jump size distribution as well as jump intensity are scaled by a constant $\left(1+\lambda_{v} \mu_{v}\right)^{-1}$, which is greater than one whenever $\psi>1, \gamma>1$. This offers a very simple and intuitive adjustment from the objective to the risk-neutral measure.

\subsection{Dividends}

We consider a stock whose perpetual dividend stream follows

$$
d \ln D_{t}=\phi d \ln C_{t}+\sigma_{d} \sqrt{V_{t}} d W_{d, t},
$$

where the parameter $\phi$ can be interpreted as a "consumption leverage" parameter or the OLS slope coefficient obtained by regressing $d \ln D_{t}$ on $d \ln C_{t}$. Whenever $\phi>1$, we can think of the corporate dividends as being a levered position on total consumption output. The idea of dividends as a levered position on consumption is useful in reconciling the low consumption volatility with high volatilities of corporate earning and dividends. The term $\sigma_{d} \sqrt{V_{t}} d W_{d, t}$ represents asset specific noise which is not priced in equilibrium.

It is straightforward to recover an exact pricing formula for the price of a stock, as in equation (3.3). However, in order to recover exponential affine stock price 
dynamics, we log-linearize stock returns, so that the equilibrium price-dividend ratio can be written as $\exp \left(A_{d}+B_{d, v}^{\prime} V_{t}\right)$. Equations (3.5) become

$$
\begin{aligned}
\left.-\kappa_{v}\left[(\theta-1) k_{1} B_{v}+k_{1, d} B_{d, v}\right)\right]-(\theta-1)\left(1-k_{1}\right) B_{v}-\left(1-k_{1, d}\right) B_{d, v} \\
+\frac{1}{2}\left[(\gamma-\phi)^{2}+\sigma_{d}^{2}+\sigma_{v}^{2}\left((\theta-1) k_{1} B_{v}+k_{1, d} B_{d, v}\right)^{2}\right] \\
+l_{1}\left[\left(1-\frac{\mu_{v}(\theta-1) k_{1} B_{v}}{\nu}-\frac{\mu_{v} k_{1, d} B_{d, v}}{\nu}\right)^{-\nu}-1\right]=0
\end{aligned}
$$

which can be solved for $B_{d, v}$ with similar caveats about multiple roots as in the previous section.

The dividend process follows

$$
\begin{aligned}
d \ln D_{t} & =\phi d \ln C_{t}+\sigma_{d} \sqrt{V_{t}} d W_{d, t}^{Q} \\
& =\phi\left(\mu-\gamma V_{t}\right) d t+\phi \sqrt{V_{t}} d W_{c, t}^{Q}+\sigma_{d} \sqrt{V_{t}} d W_{d, t}^{Q}
\end{aligned}
$$

under the risk-neutral measure. It is now straightforward to show that the stock price, which is given by $\ln P_{t}=\ln D_{t}+\left(A+B_{d, v} V_{t}\right)$, evolves according to

$$
\begin{aligned}
d \ln P_{t}=[\phi( & \left.\left.\mu-\gamma V_{t}\right)+B_{d, v}\left(\kappa_{v}\left(\bar{V}-V_{t}\right)-\lambda_{v} \sigma_{v}^{2} V_{t}\right)\right] d t \\
& +\sigma_{d} \sqrt{V_{t}} d W_{d, t}^{Q}+\phi \sqrt{V_{t}} d W_{c, t}^{Q}+B_{d, v} \sigma_{v} \sqrt{V_{t}} d W_{v, t}^{Q}+B_{d, v} \xi_{V}^{Q} d N_{t}^{Q}
\end{aligned}
$$

under the risk-neutral measure. In particular, the variation in stock price is generated by the Brownian motion shocks in dividends, consumption and market volatility as well as variance jumps. For a reasonable calibration of parameter values, the modelimplied stock price volatility is greater than the consumption volatility $V_{t}$. In fact, it is easy to generate $15-20 \%$ stock price volatility while keeping the aggregate consumption growth variation close to the historical estimates of $2-3 \%$. Therefore, our model can account for an excessive volatility of financial variables relative to the economic fundamentals.

As the conditional variance of log price is proportional to $V_{t}$, the conditional correlation between log price and its variance is given by $\operatorname{Corr}_{t}\left(d \ln P_{t}, d V_{t}\right)$. The latter is equal to

$$
\operatorname{Corr}_{t}\left(d \ln P_{t}, d V_{t}\right)=B_{d, v} \sqrt{\frac{\sigma_{v}^{2} V_{t}+\mu_{v}^{2} \nu^{-1}\left(l_{0}+l_{1} V_{t}\right)}{V_{t}\left(\sigma_{d}^{2}+\phi^{2}+B_{d, v}^{2} \sigma_{v}^{2}\right)+B_{d, v}^{2} \mu_{v}^{2} \nu^{-1}\left(l_{0}+l_{1} V_{t}\right)}},
$$

where $l_{0}, l_{1}$ and $\mu_{v}$ can be under either the physical or risk-neutral measure. Thus, the correlations of the stock price with volatility are different under the objective $P$ and risk-neutral measure $Q$. The difference in $P$ and $Q$ correlation is driven by the magnitude of the jump risk premium. In our model, the jump risk premium increases 
uniformly in the risk aversion parameter $\gamma$. Thus, larger values of $\gamma$ generate a larger dispersion in the correlation for the two measures. This effect is illustrated in Figure 4.1 .

[Figure 1 about here.]

Notice that the correlation is increasing (in absolute value) in the parameter $B_{d, v}$. This parameter typically takes on negative values for $\psi, \gamma>1$. This implies that the correlation in (4.13) approaches negative one for large values of $\gamma$ and $\psi$. Figure 4.1 illustrates this effect. The figure also shows that the correlation under both the objective and the risk-neutral measures becomes more negative when risk aversion increases. The correlation is less negative for all values of $\gamma$ when the value of the idiosyncratic dividend noise term, $\sigma_{d}$, is higher, illustrating that individual stocks (which contain a larger fraction of idiosyncratic noise) exhibit less pronounced volatility/stock price correlation than do equity indices.

The negative sign shows that increased macroeconomic uncertainty leads to lower equilibrium stock valuations. Correlations are higher in absolute value under the risk-neutral measure, and the difference between the two measures increases with the risk aversion coefficient. This is an important observation, because researchers who attempt to fit reduced form no-arbitrage models to option price data often find that the magnitude of the stock/price volatility correlation well exceeds the correlations estimated from the actual stock price and volatility estimates. For example, Bakshi, Cao and Chen (1997) calibrate jump diffusion models and find option implied correlations in the -0.6 to -0.8 range. This is generally outside the range found in returns data.

Andersen, Benzoni and Lund (2002) estimate the correlation to be in the -0.5 to -0.6 range. Eraker, Johannes and Polson (2003) estimate it to be in the -0.4 to -0.5 range. Eraker (2004) fits various no-arbitrage jump diffusion models to both returns data and joint data on options and returns and finds that the correlations are greater in magnitude when option data is included. A difference of about ten percentage points in the $P$ and $Q$ correlations is consistent with a risk aversion, $\gamma$, of about nine in our model.

The equity premium in our economy is

$$
\begin{array}{r}
\frac{1}{d t} E_{t} d \ln R_{d, t}-r_{t}=\left(\gamma \phi+\lambda_{v} k_{1, d} B_{d, v} \sigma_{v}^{2}-\frac{1}{2}\left(\sigma_{d}^{2}+\phi^{2}+k_{1, d}^{2} B_{d, v}^{2} \sigma_{v}^{2}\right)\right) V_{t}+ \\
\left(k_{1, d} B_{d, v} \mu_{v}+\varrho\left(-\lambda_{v}\right)-\varrho\left(k_{1, d} B_{d, v}-\lambda_{v}\right)\right) l\left(V_{t}\right) .
\end{array}
$$

The first bracket contains a standard CRRA risk premium $\gamma \phi V_{t}$ which will be present in a model with no jumps or time-varying stochastic volatility. The second component, $\lambda_{v} k_{1, d} B_{d, v} \sigma_{v}^{2} V_{t}$, captures the compensation for "small" volatility shocks $d W_{V, t}$, while 
the third term gives an (unimportant) quadratic variation adjustment due to the fact that we work with log, rather than level returns. In the same way, the second bracket contains the risk premium due to "large" volatility jumps $d N_{t}$ and the corresponding Ito's adjustment due to jumps in log returns.

With power utility preferences, expected excess returns do not contain the compensation for Brownian and jump volatility shocks. On the other hand, the volatility and jump compensation terms could be large in our model if $B_{d, v}$ and $\lambda_{v}$ are large. While a careful estimation of model parameters is beyond the scope of this paper, it is interesting to note that the parameter values used to generate the implied volatility graphs reported below generate an equity premium that ranges between one and twelve percent per annum. Thus, our model could potentially resolve the equity premium puzzle of Mehra and Prescott (1985). We note here that large equity premium and low risk-free rates is a well documented feature of long run risk models. Eraker (2006) studies a three factor model in which the equity premium puzzle can be resolved with small values of the risk aversion parameter $\gamma$. The presence of volatility jumps in this model significantly increases the equity premium. It is noticeable that our model can generate large equity premium even without the "long run risk" factor that captures time-variation in expected consumption growth, as is the case in the Bansal and Yaron (2004) and Eraker (2006) models.

\subsection{Price Patterns}

In the following we discuss key properties of option prices computed in our model. The standard measure of empirical patterns in option data is the options implied volatility. It is well known that the implied volatility of index options is convex over different strikes. To examine if our model can generate similar patterns, we compute implied volatilities by equating the theoretical model prices to the ones in the Black \& Scholes model using our model solutions for the dividend yields and interest rates.

In Figure 4.2 we plot the implied volatility as a function of the strike price and maturity. The two surface plots are generated for low and high initial values of the volatility $V_{t}$. The implied volatility patterns are fairly typical of those computed in no-arbitrage models which feature high negative correlation between volatility shocks and prices. The implied volatility has a more pronounced U shape at short maturities, and flattens out at the long end. Evidence of negative skewness is evident even for long maturity contracts.

[Figure 2 about here.]

Figure 4.3 depicts the implied volatility of our theoretical model prices computed over different values of risk aversion $\gamma$ and with a fixed value of $\psi=4$. There are two 
main effects of increasing the level of risk aversion. First, the stock prices become more volatile on average. This is reflected by an upward shift in implied volatility for higher values of $\gamma$. Second, and more interesting, the convexity of the curve is much more pronounced for higher values of $\gamma$, reflecting a more heavily tailed equilibrium stock price distribution. Jumps to the volatility process always result in (negative) jumps in the stock price, as can be seen from equation (4.12). The negative price reactions to a jump in volatility can be significant, as evidenced by the increasing prices of far out-of-the-money put options in Figure 4.3. Therefore, endogenizing the stock price in an economy where the volatility may increase suddenly can explain the high crash insurance premiums offered by out-of-the-money put options.

[Figure 3 about here.]

Does CRRA utility deliver implied volatility graphs that mimic those of the general Epstein-Zin model? Recall that the CRRA model obtains as a special case by imposing the constraint $\psi=1 / \gamma$. We impose this condition in computing implied volatilities in figure 4.4. The main difference between the two equilibrium specifications is that CRRA model produces implied volatility curves that imply positive conditional skewness in the risk-neutral stock price distribution, as can be seen from the fact that contracts with high strikes carry a higher premium. This positive skewness is attributable to the fact that the parameter $B_{d, v}$ is positive, indicating a positive volatility-return correlation in 4.13. For Epstein-Zin preferences, $B_{d, v}$ is negative, which yields a negative volatility-return correlation and thus a negatively skewed stock price density. Thus, Epstein-Zin based equilibrium option prices tend to generate implied volatility curves that are consistent with those observed empirically.

[Figure 4 about here.]

It is possible to generate very steep equilibrium implied volatility curves in our example model. Recall that the parameter $\nu$ determines the tail-behavior of the volatility jump sizes, and a small value of $\nu$ leads to heavier tails. Figure 4.5 illustrates the effect of changing $\nu$. The impact of out-of-the-money puts is significant, and prices increase uniformly with lower values of $\nu$. The case where $\nu=0.15$ illustrates that the possibility of a very severe jump in volatility has a dramatic effect on the price of OTM puts.

[Figure 5 about here.]

Our discussion so far has mostly been relevant in the context of options written on an index of stocks. Stock indices, unlike individual equities, are characterized by the 
fact that the risks are almost entirely systematic. In our model this is captured by a small value of $\sigma_{d}$ (we used 0.2 in our preceding discussion). Figure 4.6 illustrates the effect of increasing idiosyncratic risk, $\sigma_{d}$, on option prices. While the overall implied volatility increases, higher values of $\sigma_{d}$ significantly diminish conditional skewness and kurtosis as can be seen from an almost flat implied volatility curve. Increasing $\sigma_{d}$ in our model thus has an effect of just adding Gaussian noise to the stock price process. Our model can easily be augmented to allow for company specific dividend jumps. This would generate additional kurtosis, and thus additional convexity in implied volatilities of individual stock options.

[Figure 6 about here.]

\section{Concluding Remarks}

Affine class constitutes a significant and important class of asset pricing models. Affine models are typically derived under assumptions of no-arbitrage, which offers a limited ability for economic interpretation of the market prices of risks that link the objective probability measure with the risk-neutral pricing measure. Our paper suggests a way to remedy this by constructing a pricing kernel which is based on generalized preferences of Epstein and Zin (1989). Importantly, our framework offers a convenient way to link the two measures in the presence of diffusion risks and jump risks. All risk premia computations in our model are done through the specification of the three preference parameters $(\delta, \psi$ and $\gamma)$. Since market prices of risk are explicit functions of these three parameters, our model framework offers a more parsimonious way to compute equilibrium prices than the usual affine no-arbitrage models which do not restrict market prices of risk.

We set up an example model with only one state variable, variance of consumption growth driven by Brownian motion shocks and Poisson jumps, and study the equilibrium effects on the equity and option prices. Our model endogenously generates a negative correlation between shocks to the volatility process and the equilibrium stock prices. The compensation for the variance shocks is sizeable and can help to account for a high expected equity return relative to the risk-free rate. The implied volatilities are $\mathrm{U}$ shaped, with significantly higher prices for out-of-the-money puts; the smile flattens out with a decrease in the risk aversion coefficient. These effects can not be captured in traditional power utility models.

It is straightforward to include additional state variables into our framework, such as time-varying expected consumption growth, inflation, multiple volatility and jump intensity processes, etc. The model can also be confronted with macro and financial data; there are several possible empirical strategies for identification and estimation 
of the preference and cash flow dynamics parameters and evaluation of the model in and out of sample. We leave the issues of more elaborate models and empirical fit for a future research. 


\section{A Log-linearization in Continuous Time}

Let $d \ln R_{t}$ heuristically denote the continuous time $\log$ return so that $\int_{t}^{t+1} d \ln R_{s}$ define the return over the interval $(t, t+1]$. The Campbell-Shiller approximation to the discrete time return is then

$$
\int_{t}^{t+1} d \ln R_{s}=k_{0}+k_{1} v_{t+1}-v_{t}+\Delta \ln D_{t+1}
$$

where $v_{t}$ is the $\log$ price-dividend ratio, and $\Delta \ln D_{t}$ is the dividend growth rate. Equation (A.1) is exactly the same as in discrete time, as we showed in Section 2.

Now rewrite (A.1) as

$$
\begin{aligned}
\int_{t}^{t+1} d \ln R_{s} & =k_{0}+k_{1}\left(v_{t+1}-v_{t}\right)-\left(1-k_{1}\right) v_{t}+\Delta \ln D_{t+1} \\
& =\int_{t}^{t+1} k_{0} d s+k_{1} \int_{t}^{t+1} d v_{s}-\left(1-k_{1}\right) \int_{t}^{t+1} v_{t} d s+\int_{t}^{t+1} d \ln D_{s}
\end{aligned}
$$

From here we define the continuous time return $d \ln R_{t}$ :

$$
d \ln R_{t}=k_{0} d t+k_{1} d v_{t}-\left(1-k_{1}\right) v_{t} d t+d \ln D_{t}
$$

For the consumption asset, $v_{t}=A+B^{\prime} X_{t}$ and $d \ln C_{t}=\delta_{c}^{\prime} d X_{t}$, so that $d \ln R_{c, t}$ is

$$
d \ln R_{c, t}=k_{0} d t+\left[\delta_{c}+\kappa_{1} B\right]^{\prime} d X_{t}-\left(1-k_{1}\right)\left(A+B^{\prime} X_{t}\right) d t .
$$

Similar decomposition holds for a log return on a dividend asset $d \ln R_{d, t}$. 


\section{References}

Aase, K. (2002): Equilibrium Pricing in the Presence of Cumulative Dividends Following a Diffusion, Mathematical Finance 12, 173-198.

Andersen, T. G., L. Benzoni, and J. Lund (2002): An Empirical Investigation of Continuous-Time Models for Equity Returns, Journal of Finance 57, 1239-1284.

Bakshi, G., C. Cao, and Z. Chen (1997): Empirical Performance of Alternative Option Pricing Models, Journal of Finance 52, 2003-2049.

Bansal, R. (2007): Long Run Risks and Financial Markets, Working paper, Duke University.

Bansal, R., V. Khatchatrian, and A. Yaron (2005): Interpretable Asset Markets, European Economic Review 49, 531-560.

Bansal, R., D. Kiku, and A. Yaron (2006): Risks for the Long Run: Estimation and Inference, Working paper, Duke University.

Bansal, R., and I. Shaliastovich (2007): Risk and Return in Bond, Currency and Equity Markets, Working paper.

Bansal, R., and A. Yaron (2004): Risks for the Long Run: A Potential Resolution of Asset Pricing Puzzles, Journal of Finance 59, 1481-1509.

Barillas, F., L. Hansen, and T. Sargent (2006): Reinterpreting a Graph (and a Parameter) of Tallarini, Working paper.

Barndorff-Nielsen, O. E., and N. Shephard (2001): Non-Gaussian Ornstein-UhlenbeckBased Models and Some of Their Uses in Financial Economics, Journal of Royal Statistical Society 63, 167-241.

Bates, D. S. (1996): Jump and Stochastic Volatility: Exchange Rate Processes Implicit in Deutsche Mark Options, Review of Financial Studies 9, 69-107.

Bates, D.S. (2000): Post-87 Crash fears in S\&P 500 Futures Options, Journal of Econometrics 94, 181-238.

Benzoni, L., P. Collin-Dufresne, and R. S. Goldstein (2005): Can Standard Preferences Explain the Prices of Out-of-the-Money S\&P 500 Put Options? Working paper, University of Minnesota.

Campbell, J. (1993): Intertemporal Asset Pricing without Consumption Data, American Economic Review 83, 487-512.

Campbell, J. Y., A.W. Lo, and A. C. Mackinlay (1997): The Econometrics of Financial Markets. Princeton, N.J.: Princeton University Press. 
Campbell, J. Y., and R. J. Shiller (1988): Stock Prices, Earnings and Expected Dividends, Journal of Finance 43, 661-676.

Carr, P., H. Geman, D. Madan, and M. Yor (2003): Stochastic Volatility for Levy Process, Mathematical Finance 13, 345-382.

Carr, P., and D. Madan (1999): Option Pricing and the Fast Fourier Transform, Journal of Computational Finance 2, 61-73.

Chen, H. (2006): Macroeconomic Conditions and the Puzzles of Credit Spreads and Capital Structure, Working paper.

Cont, R., and P. Tankov (2004): Financial Modelling with Jump Processes. London: Chapman \& Hall.

Duffie, D., and L. Epstein (1992): Stochastic Differential Utility, Econometrica 60, 353-394.

Duffie, D., and R. Kan (1996): A Yield-Factor Model of Interest Rates, Mathematical Finance 6, 379-406.

Duffie, D., J. Pan, and K. J. Singleton (2000): Transform Analysis and Asset Pricing for Affine Jump-Diffusions, Econometrica 68, 1343-1376.

Epstein, L. G., and S. E. Zin (1989): Substitution, Risk Aversion, and the Temporal Behavior of Consumption and Asset Returns, Econometrica 57, 937-69.

Eraker, B. (2004): Do Stock Prices and Volatility Jump? Reconciling Evidence from Spot and Option Prices, Journal of Finance 59, 1367-1403.

Eraker, B. (2006): Affine General Equilibrium Models, Working paper, Duke University.

Eraker, B., M. J. Johannes, and N. G. Polson (2003): The Impact of Jumps in Returns and Volatility, Journal of Finance 53, 1269-1300.

Garcia, R., R. Luger, and E. Renault (2003): Empirical Assessment of an Intertemporal Option Pricing Model with Latent Variables, Journal of Econometrics 116, 49-83.

Hansen, L., and T. Sargent (2006): Fragile Beliefs and the Price of Model Uncertainty, Working paper.

Hansen, L. P., J. Heaton, and N. Li (2004): Consumption Strikes Back, Working paper, University of Chicago.

Heston, S. (1993): Closed-Form Solution of Options with Stochastic Volatility with Application to Bond and Currency Options, Review of Financial Studies 6, 327-343. 
Hull, J., and A. White (1987): The Pricing of Options with Stochastic Volatilities, Journal of Finance 42, 281-300.

Lewis, A. (2000): Option Valuation under Stochastic Volatility. Finance Press.

Liu, J., J. Pan, and T. Wang (2005): An Equilibrium Model of Rare-Event Premia and its Implication for Option Smirks, Review of Financial Studies 18, 131-164.

Madan, D., P. Carr, and E. Chang (1998): The Variance Gamma Process and Option Pricing, European Finance Review 2, 79-105.

Maenhout, P. J. (2004): Robust Portfolio Rules and Asset Pricing, Review of Financial Studies 17, 951-983.

Mehra, R., and E. Prescott (1985): The Equity Premium: A Puzzle, Journal of Monetary Economics 15, 145-161.

Schroder, M., and C. Skiadas (1999): Optimal Consumption and Portfolio Selection with Stochastic Differential Utility, Journal of Economic Theory 89, 68-126.

Shaliastovich, I., and G. Tauchen (2006): Pricing Implications of Stochastic Volatility, Business Cycle Time Change, and Non-Gaussianity, Working paper, Duke University.

Tallarini, T. (2000): Risk-Sensitive Real Business Cycles, Journal of Monetary Economics 45(3), 507-532.

Tauchen, G. (2005): Stochastic Volatility in General Equilibrium, Working paper, Duke University.

Weil, P. (1989): The Equity Premium Puzzle and the Risk Free Rate Puzzle, Journal of Monetary Economics 24, 401-421 


\section{Notes}

${ }^{1}$ For robust empirical evidence on the magnitude of the IES and the asset pricing implications in equity, bond and currency markets refer, for instance, to Bansal, Khatchatrian and Yaron (2005), Eraker (2006) and, Bansal and Shaliastovich (2007).

${ }^{2}$ Other approximations are possible - see for example Hansen, Heaton and Li (2004), Benzoni, Collin-Dufresne and Goldstein (2005).

${ }^{3}$ These results apply under certain technical existence and integrability conditions (see, e.g. Cont and Tankov 2004 ). 


\section{Tables and Figures}




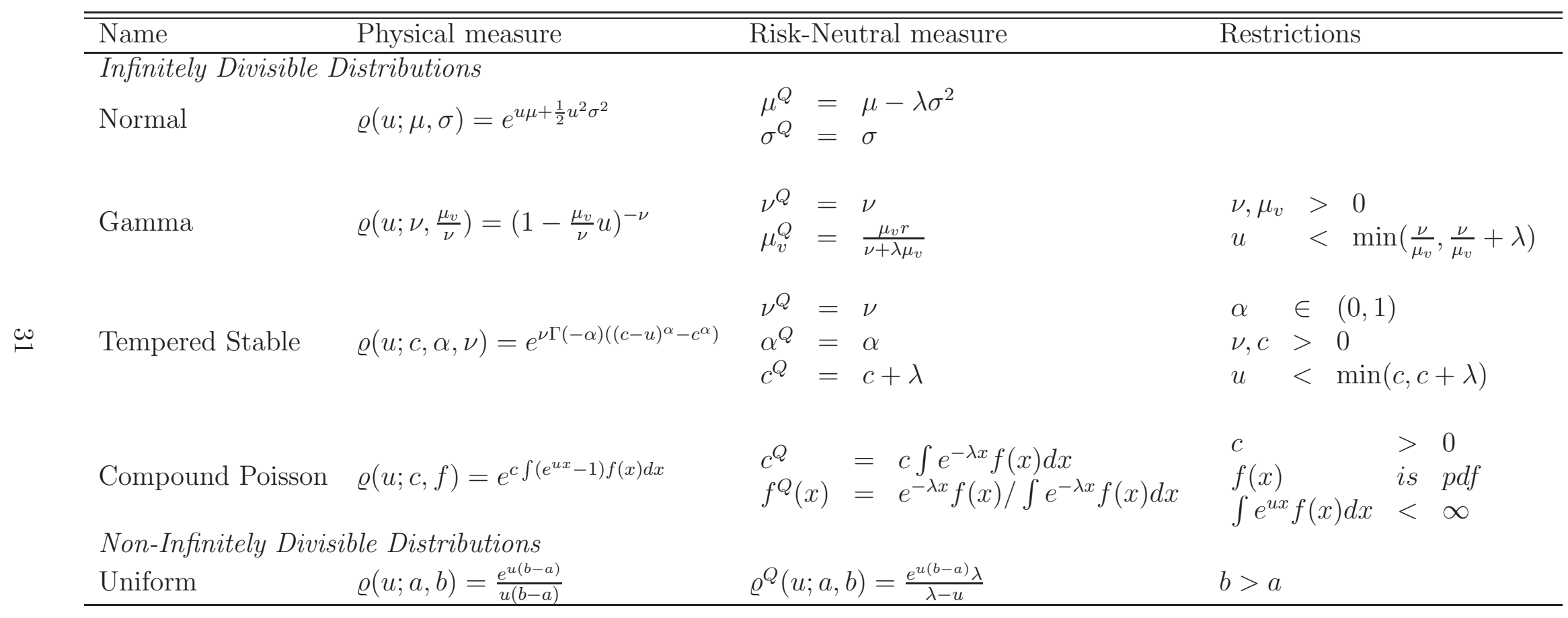

Table 1: The risk-neutral adjustment to the jump size distribution. 


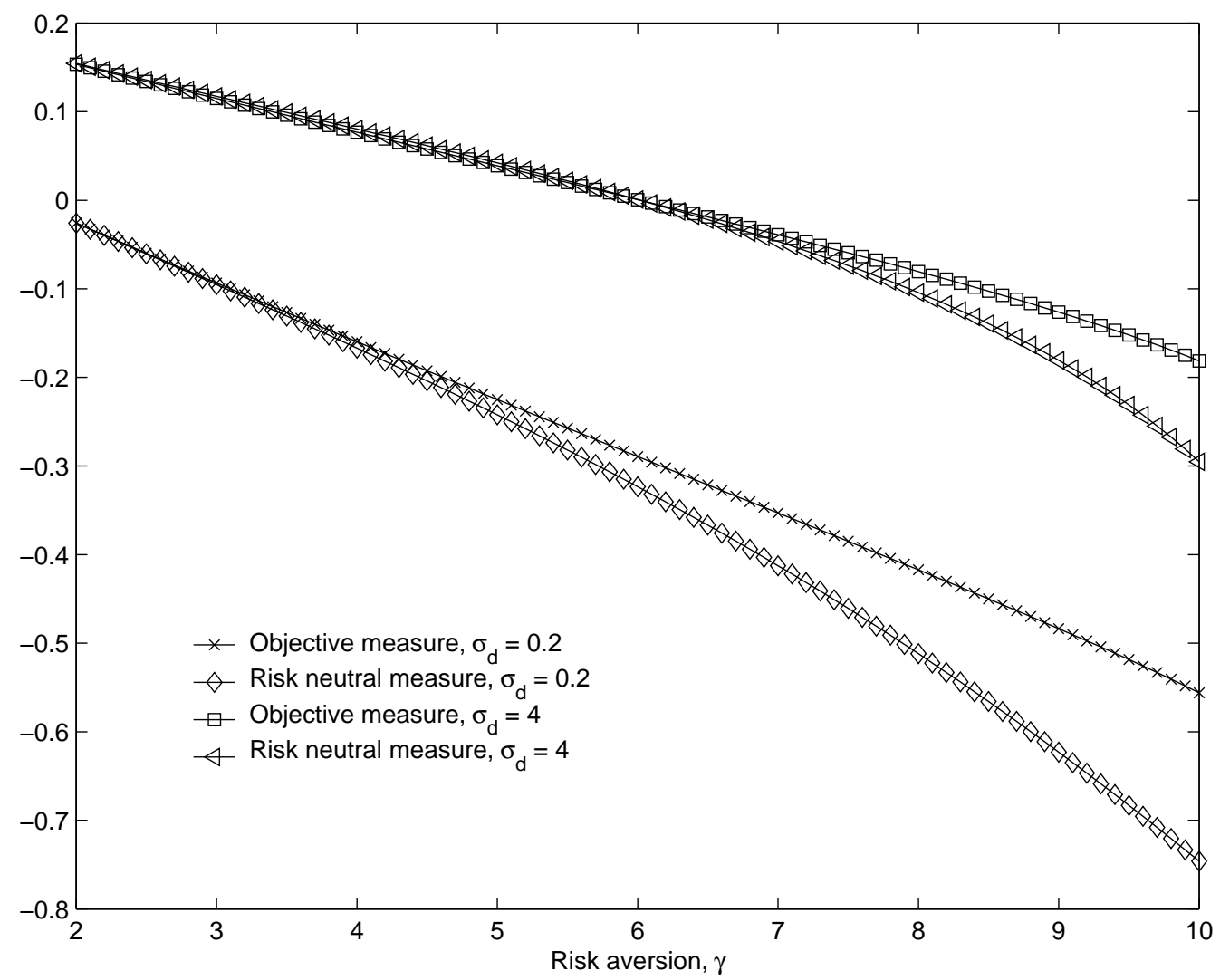

Figure 4.1: Correlation between the innovations in log stock price and market variance as a function of risk aversion $\gamma$. 


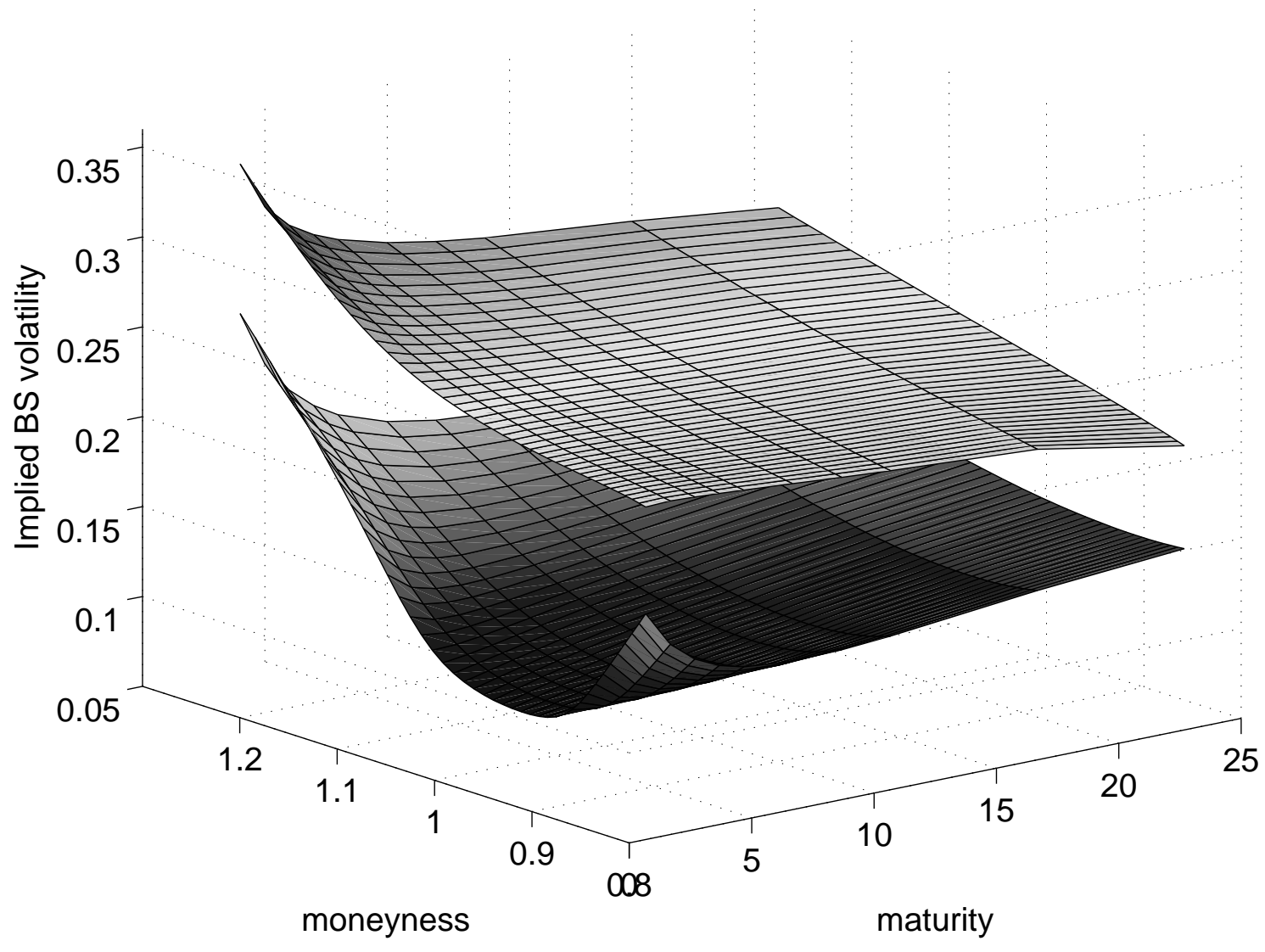

Figure 4.2: Implied volatility across strikes and maturities. The upper (lower) surface plot corresponds to high (low) initial volatility $V_{t}$. 


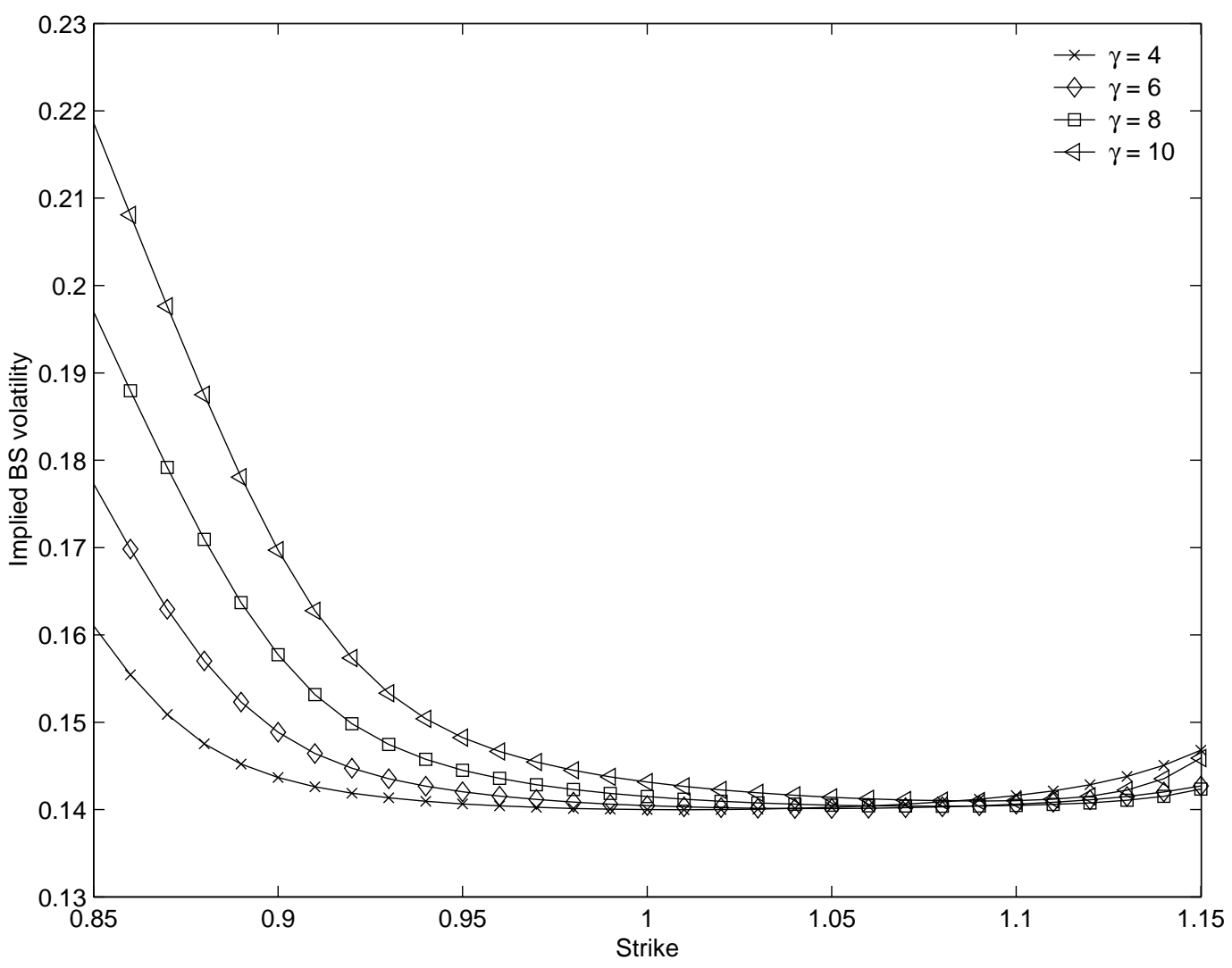

Figure 4.3: Implied volatility for different levels of risk aversion $\gamma$. 


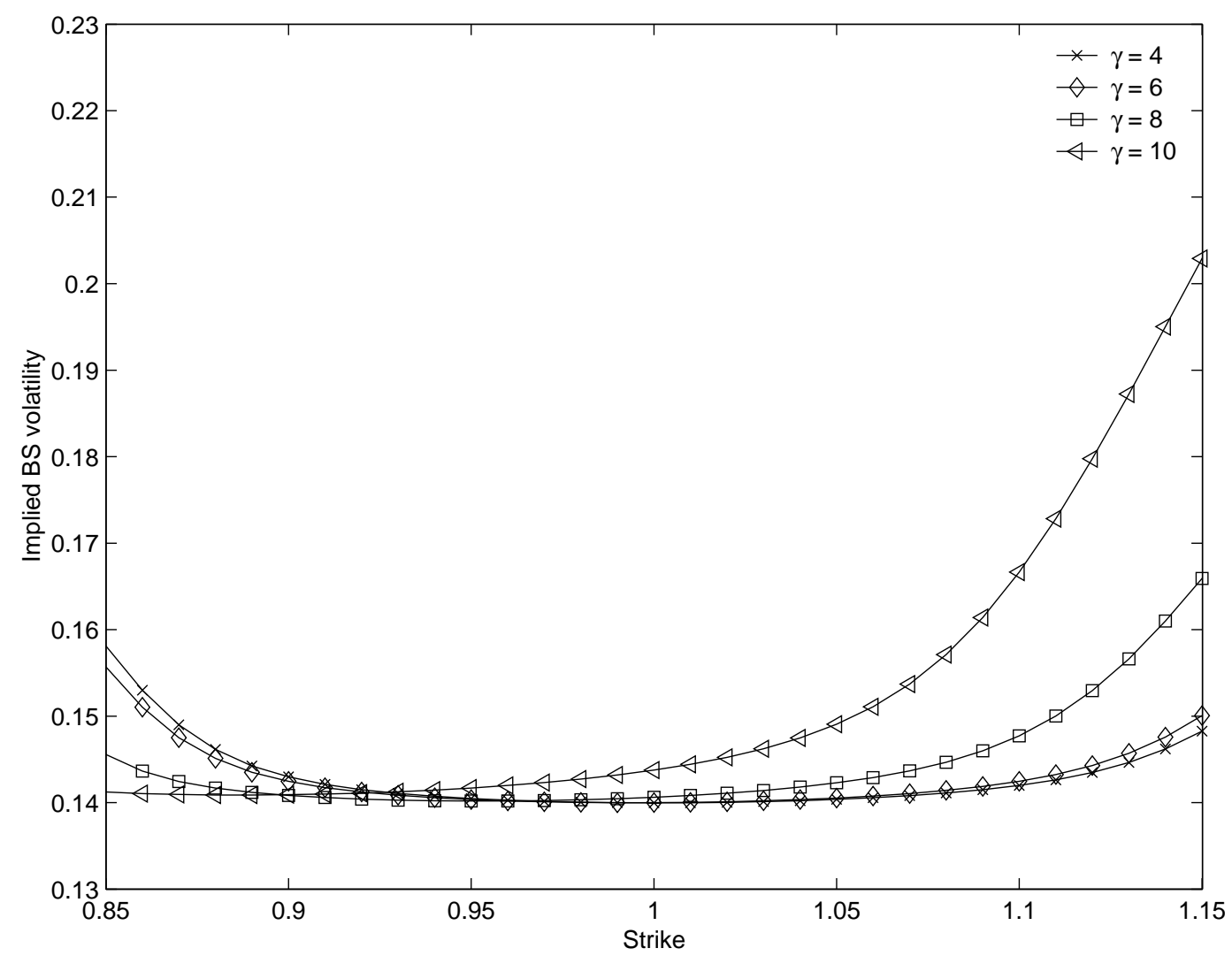

Figure 4.4: Implied volatility for different levels of risk aversion $\gamma$ in the case of CRRA utility. 


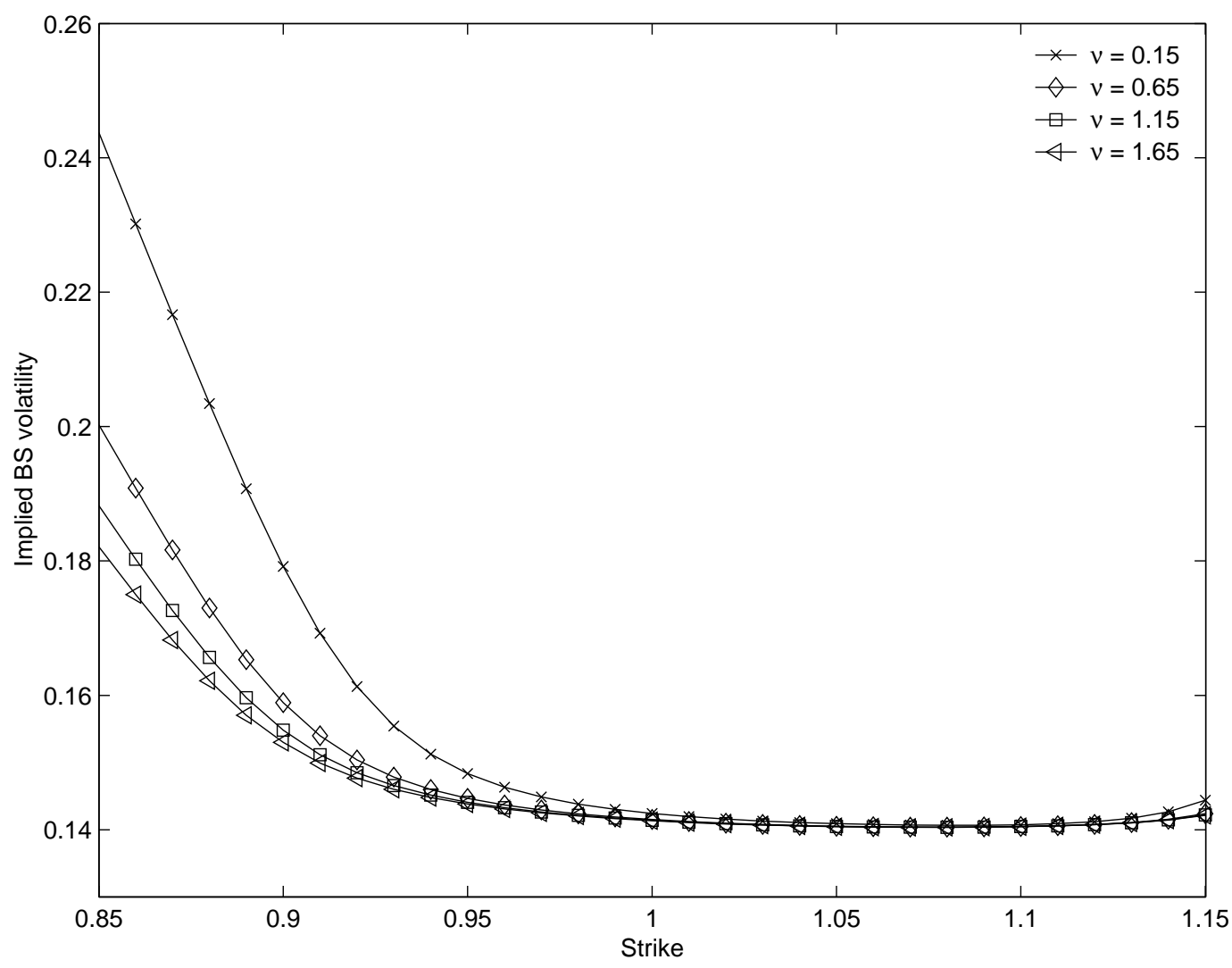

Figure 4.5: Implied volatility for different levels of volatility jump tail-thickness $\nu$. 


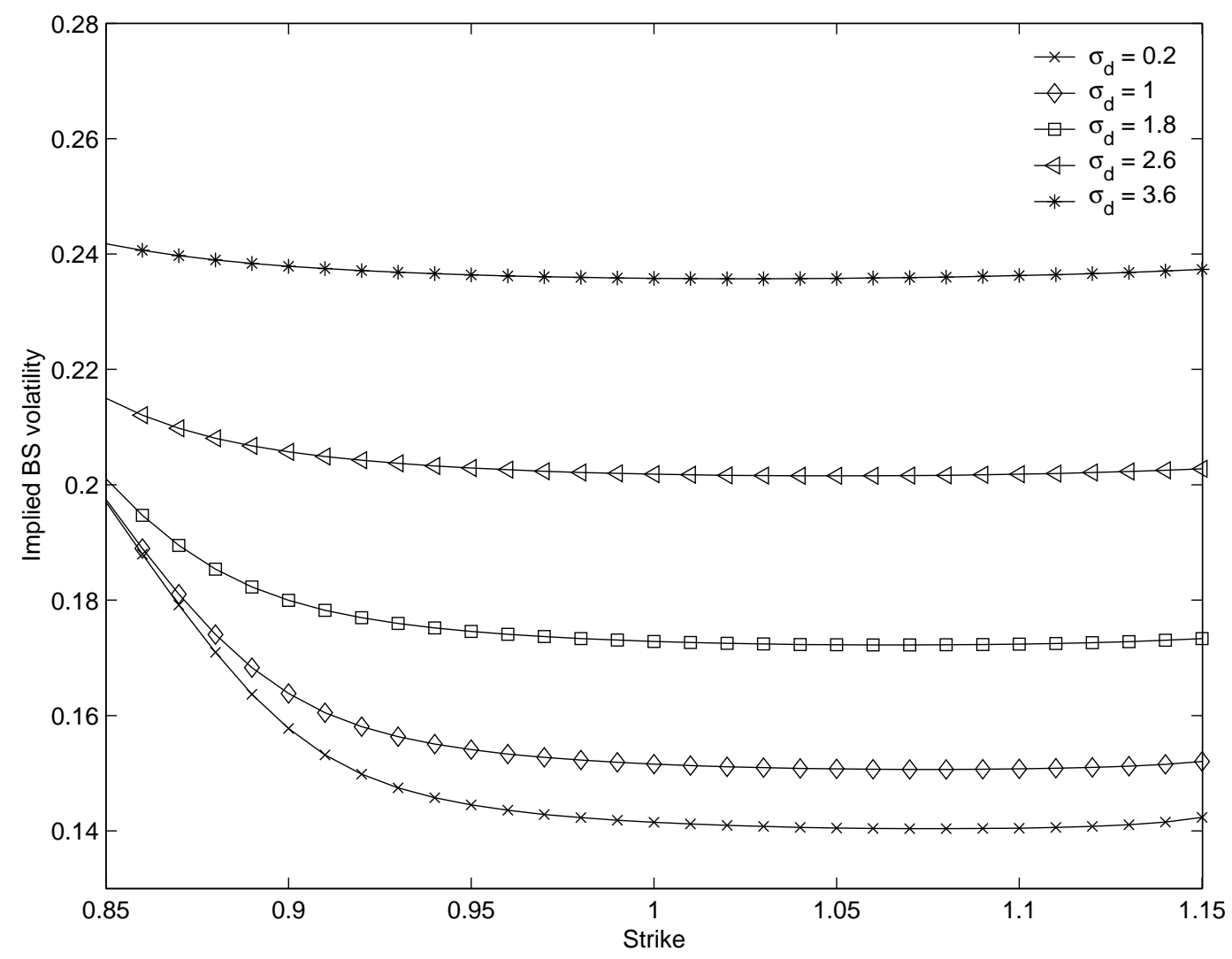

Figure 4.6: Implied volatility for different levels of idiosyncratic risk $\sigma_{d}$. 